\title{
Variable Angle Spectroscopic Ellipsometry and Electron Energy-Loss Spectroscopy
}

Jessica A. Alexander ${ }^{1}$, Frank J. Scheltens ${ }^{1}$, Lawrence F. Drummy ${ }^{2}$, Michael F. Durstock ${ }^{2}$, James B. Gilchrist $^{3}$, Sandrine E.M. Heutz ${ }^{3}$, and David W. McComb ${ }^{1}$

${ }^{1 .}$ Center for Electron Microscopy and Analysis, Department of Materials Science and Engineering, The Ohio State University, College Road, Columbus OH 43210, United States

2. Materials and Manufacturing Directorate, Air Force Research Laboratory, WPAFB, Ohio 45433, United States

3. Department of Materials, Imperial College London, SW7 2AZ, United Kingdom

Electron energy-loss spectroscopy (EELS) in the scanning transmission electron microscope (STEM) has considerable potential for investigation of interfaces in organic photovoltaic (OPV) devices. In particular, the low-loss region of the EEL spectrum can be used to obtain the complex dielectric function of the material. The complex dielectric function in turn allows us to distinguish between single electron transitions and collective excitations. Spatial mapping of the single electron transitions can then be used to learn about the chemistry and bonding in the vicinity of interfaces between the acceptor and donor interfaces in the OPV samples [1].

In order to validate EELS measurements of organic samples, the technique of variable angle spectroscopic ellipsometry (VASE) has been used. In VASE, a light beam is directed onto the sample, and information related to phase shifts and amplitude changes of the incident beam upon reflection off of the sample is collected and analyzed [2]. Similar to EELS, it is possible to determine the energydependent refractive index and extinction coefficient, as well as the energy-dependent real and imaginary dielectric components of the complex dielectric function. Since the source is a light beam, it is possible to assume that the sample undergoes little or no beam damage. As a result, the data collected using VASE can be compared to the data collected using EELS in order to substantiate the results from EELS so that it is possible to determine if the organic samples have undergone any damage in the electron microscope.

This analysis has been done for four different organic materials of interest: copper phthalocyanine (CuPc), fullerene- $\mathrm{C}_{60}$, poly(3-hexylthiophene) (P3HT), and [6,6] phenyl $\mathrm{C}_{61}$ butyric acid methyl ester (PCBM). Thin films of $\mathrm{CuPc}, \mathrm{C}_{60}$, and PCBM were prepared by thermal evaporation of the organic material onto freshly cleaved rock salt substrates $\left(\mathrm{KCl}\right.$ for $\mathrm{CuPc}$ and $\mathrm{PCBM} ; \mathrm{NaCl}$ for $\left.\mathrm{C}_{60}\right)$ using an organic evaporation chamber in an argon-filled glove box. The deposited films were of thicknesses of 50 $\mathrm{nm}$ or less so that they would be suitable for imaging and spectroscopy in the scanning transmission electron microscope (STEM). For ellipsometry measurements, these films were left as prepared on the rock salt substrates, but for EELS measurements, the thin films were floated off by dissolution of the substrates in distilled water and collected on lacey carbon coated TEM grids. Figure 1 shows TEM images of $\mathrm{CuPc}$ and $\mathrm{C}_{60}$ thin films prepared in this manner. Lattice fringes in the TEM image of $\mathrm{CuPc}$ confirm that the deposited film has crystalline structure. Thin films of P3HT were prepared by spincoating a solution of $\mathrm{P} 3 \mathrm{HT}$ and dichlorobenzene onto $\mathrm{KCl}$ substrates.

For example, a comparison of the real dielectric functions obtained from VASE and EELS measurements on $\mathrm{CuPc}$ is shown in Figure 2. One sample (denoted as CuPc/PTCDA) consisted of evaporated $\mathrm{CuPc}$ on $\mathrm{KCl}$ with an interlayer of PTCDA and a second sample (denoted as $\mathrm{CuPc}$ ) consisted 
of evaporated $\mathrm{CuPc}$ on $\mathrm{KCl}$. The datasets obtained were in good agreement with previously reported data for evaporated $\mathrm{CuPc}$ on $\mathrm{Si}$ [3]. The EELS data obtained from the $\mathrm{CuPc}$ and $\mathrm{CuPc} / \mathrm{PTCDA}$ samples are also in good agreement with the VASE data. This correlation validates the use of STEM-EELS measurements for OPV studies and confirms that such data can be obtained without electron beam damage of the molecular structure.

\section{$\underline{\text { References }}$}

[1] R.F. Egerton in "Electron Energy-Loss Spectroscopy in the Electron Microscope, Third Edition," (Springer, New York).

[2] H.G. Tompkins \& E.A. Irene in "Handbook of Ellipsometry," (Springer, 2005).

[3] D. Datta, V. Tripathi, et al., Thin Solid Films 516 (2008), 7237.

[4] The authors acknowledge the Air Force Office of Scientific Research and the Air Force Research Laboratory (AFRL) Materials and Manufacturing Directorate for funding, Kurt Eynik and Luke Bissel from AFRL for technical support, and The Ohio State University Center for Electron Microscopy and Analysis for technical support.
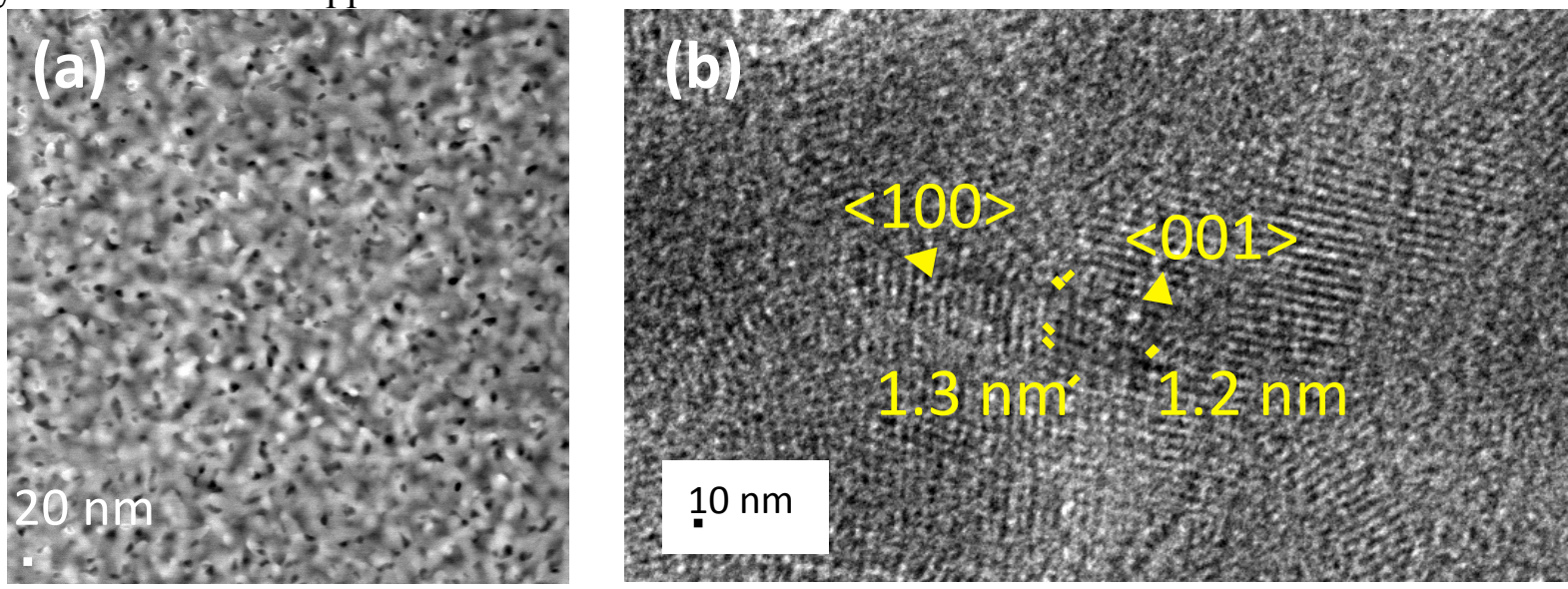

Figure 1. (a) HAADF STEM image of evaporated CuPc film. (b) indexed HRTEM image of evaporated $\mathrm{CuPc}$ film.

口

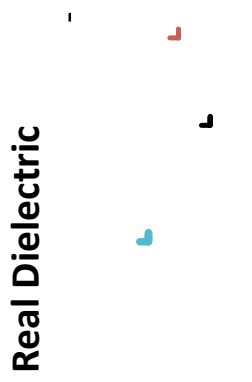

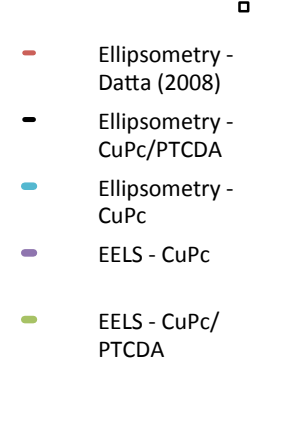

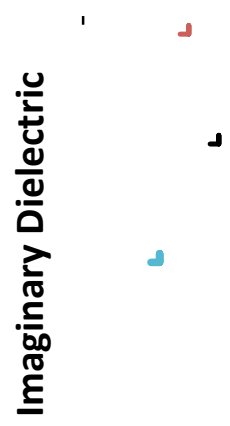

6.0
0.0
1.0

\section{$2.0 \quad 3.0 \quad 4.0 \quad 5.0$
Energy (eV)}

Figure 2. Comparisons of the real and imaginary dielectric functions for $\mathrm{CuPc}$. 